\title{
Orofacial muscles activity in children with swallowing dysfunction and removable functional appliances
}

\author{
Giuseppe Messina (1,2), Valerio Giustino (3), Francesco Martines (4,5), Serena Rizzo \\ (6), Alessio Pirino (7), Fabio Scoppa (8) \\ (1) Department of Psychology, Educational Science and Human Movement, University of \\ Palermo, Palermo, Italy; (2) Posturalab Italia Research Institute, Palermo, Italy; (3) PhD \\ Program in Health Promotion and Cognitive Sciences, University of Palermo, Palermo, Italy; \\ (4) Istituto Euromediterraneo di Scienza e Tecnologia - IEMEST, Palermo, Italy; (5) Bio.Ne.C. \\ Department, Audiology Section, University of Palermo, Palermo, Italy; (6) Di.Chir.On.S. \\ Department, Physical Medicine and Rehabilitation, University of Palermo, Palermo, Italy; (7) \\ Department of Biomedical Sciences, Division of Human Anatomy, University of Sassari, Sassari, \\ Italy; (8) Faculty of Medicine and Dental Surgery, Sapienza University of Rome, Rome, Italy
}

This article is distributed under the terms of the Creative Commons Attribution Noncommercial License (CC BY-NC 4.0) which permits any noncommercial use, distribution, and reproduction in any medium, provided the original author(s) and source are credited.

\begin{abstract}
Swallowing dysfunction is a frequent disorder among children and refers to an altered tongue posture and abnormal tongue movement during swallowing. Removable functional appliance is one of the treatments applied by dentistry to correct this disorder. The aim of this study was to evaluate any differences on orofacial muscles activity in children with swallowing dysfunction with and without removable functional appliances. 68 children were eligible for the study and divided into the orthodontic group (OG) and the no-orthodontic group (NO-OG). Both groups performed a dental occlusion-class evaluation, a swallowing function test and a myoscan analysis in order to measure perioral forces (i.e. tongue extension force, lip pressure, masseter contraction force). Our results showed a significant difference $(\mathrm{P}=0.02)$ between $\mathrm{OG}$ and NO-OG for the tongue extension force, whereas no significant differences $(\mathrm{P}>0.05)$ were found for the other parameters. Our findings suggest that children with swallowing dysfunction and removable functional appliance show orofacial muscles activity within the range of reference values (except for the lip pressure). However, we hypothesize that orthodontic treatment can achieve more effective results with integration of myofunctional therapy.
\end{abstract}

Key Words: Orofacial muscles, perioral muscles, muscles activity, swallowing dysfunction.

Eur J Transl Myol 29 (3): 246-250, 2019

The stomatognathic system is the oral apparatus in which all the structures that compose it (i.e. maxilla and mandible bones, temporomandibular joints and the ligaments associated with them, masticatory muscles and dento-periodontal structures) work synergically allowing the oral functions of chewing, swallowing and phonation. ${ }^{1,2}$ It is widely known that the interaction between this functional complex and the neck and trunk muscles affects the cranio-cervical-mandibular system and, furthermore, several authors have been investigated the influence of this apparatus on features as strength, flexibility and body posture reporting contrasting findings. ${ }^{3-7}$ In this context, research suggests that biomechanical or functional disturbances of the stomatognathic system (e.g. malocclusions, temporomandibular disorders, chewing or swallowing dysfunctions) have a direct impact on masticatory muscles inducing musculature asymmetries and modifying head posture and, through the muscle chains, adaptation mechanisms on body posture. ${ }^{8,9}$ Swallowing dysfunction is characterized by the abnormal movement of the tongue that pushes anteriorly or laterally against the dental arches during swallowing. ${ }^{10}$ In particular, the percentage of swallowing disorders in children with normal development is between $25 \%$ and $35 \% .^{11}$ As malocclusions produce modifications on masticatory muscles, likewise, in subjects with swallowing dysfunction the tongue contraction strength is altered and generates changes in electrical activity of other perioral muscles. ${ }^{12,13}$ Moreover, it would seem that function of orofacial muscles, as masseter, temporal and orbicularis oris, influences craniofacial growth and the shape of the 
face for this reason may also affect malocclusions. ${ }^{14-16}$ Similarly, swallowing dysfunctions are related with facial morphology having a direct influence on malocclusions. ${ }^{17,18}$ In order to correct any malocclusion or oral dysfunction as swallowing dysfunction, various types of functional orthodontic appliances are used in dentistry. ${ }^{19}$ Scientific literature reported that, per se, functional appliances produce intraoral forces modulating postural and functional activity of masticatory muscles. ${ }^{20,21}$ During the treatment it is essential to monitor the activity of the masticatory muscles both for the success of the orthodontic correction and for the evaluation of postural changes. ${ }^{20,22}$ Amongst the non-invasive methods in order to evaluate facial muscles activity, surface electromyography represents the most applied technique also in dentistry. ${ }^{14}$ Moreover, some of the non-invasive methods employed to assess perioral muscles strength are myoscan analysis and dynamometry. ${ }^{10}$ The aim of this study was to evaluate any differences on perioral muscles forces (i.e. tongue, lip muscles and masseter muscles) in children with swallowing dysfunction with and without removable functional appliances. The novelty of our study was to investigate the influence of removable functional appliances in these children's muscles.

\section{Materials and Methods}

\section{Participants}

A non-randomized controlled trial was adopted in a number of 68 children with swallowing dysfunction. 36 males and 32 females was eligible for the study and divided into: 1) the orthodontic group (OG) formed by children with removable functional appliance at the moment of the recruitment and children who had received functional orthodontic treatment prior to the recruitment $(n=44$; male $=23$; female $=21$; mean age: $8.75 \pm 1.12$ years); 2 ) the no-orthodontic group (NO-OG) composed by children without functional appliance at the moment of the recruitment and who have never had a functional orthodontic appliance $(n=24 ;$ male $=13$; female $=11$; mean age: $8.08 \pm 1.41$ years). To be included in the study, participants had to respect the following inclusion criteria: 1) aged 6-10 years; 2) presence of swallowing dysfunction; 3) removable functional appliance at the moment of the enrollment or previously treated with functional appliance (for the OG group). Participants were not considered eligible for the study in the presence of: 1) temporomandibular dysfunctions; 2) muscular disorders; 3) previous facial surgeries; 4) growth diseases $(n=1)$.

Both groups performed the protocol assessment including: 1) dental occlusion-class evaluation; 2) swallowing function test using Payne's technique; 3) myoscan analysis in order to measure perioral forces (i.e. tongue extension force, lip pressure, masseter contraction force).

All participants were recruited from a dental practice in Rome, Italy. Since minors, parents or guardians have provided written consent to participate in this study. The study was approved by a local ethics committee (no. $1 / 2018$ ) in conformity with criteria for the use of persons in research as defined by the Declaration of Helsinki.

\section{Dental occlusion-class evaluation}

For the dental occlusion-class evaluation all the participants were assessed in sitting position with neutral head posture and in maximum dental intercuspation. Dental occlusion-class was established according to Angle's classification based on the relationship between the first molars of the dental arches. ${ }^{23}$ In all the sample, 32 participants were in first class (in which the mesiobuccal cusp of the first molar of the maxillary bone is in relationship with the mesiobuccal groove of the first molar of the mandibular bone) and 36 participants were in second class (in which the first molar of the maxillary bone is in relationship mesially with the first molar of the mandibular bone). As concern the groups, the OG group showed 19 participants in first class and 25 participants in second class, while, the NO-OG group showed 13 participants in first class and 11 participants in second class.

\section{Swallowing function test}

The swallowing function test was performed using Payne's technique. ${ }^{24}$ During the assessment each participant was in sitting position with the head in neutral posture and against the wall and feet on the floor. A fluorescein solution was placed on the anterior and lateral edges of the tongue of each participant and subsequently were asked to perform a swallowing action. Finally, using a black light (Payne Lamp model; OMFT; Maartensdijk, De Bilt, Utrecht, The Netherlands) we were able to determine the tongue contact areas in the oral cavity during swallowing.

\section{Myoscan analysis}

As the abovementioned test, each participant was in sitting position with the head in neutral posture and against the wall and feet on the floor. In order to evaluate the orofacial muscles activity was employed the myoscan analysis using the Myoscanner 160-B (OMFT; Maartensdijk, De Bilt, Utrecht, The Netherlands). The device measures tongue extension force, lip pressure and masseter contraction force. For each muscle three trials of maximal isometric force were performed with threeminute rest between trials. The best value, expressed in pounds units, was used for statistical analysis. Agematched reference values are in Table $1 . .^{10,25}$

The standardized protocol of myoscan analysis, ${ }^{10}$ was:

- for the tongue extension force, participants were asked to push the tongue forward against the tongue-specific probe plate as strong as possible.

- for the lip pressure, participants, with the mouth closed, were asked to stretch lips forward and push against the probe, without any plate, as strong as possible. 
Table 1. Reference values age-matched of perioral muscles forces

\begin{tabular}{lcc}
\hline & $\mathbf{4 - 1 0}$ years & 10 years \\
\cline { 2 - 3 } Tongue extension force & $0.6-0.8$ & $0.8-1.2$ \\
Lip pressure & $0.2-0.6$ & $0.6-0.8$ \\
Masseter contraction & $0.4-0.6$ & $0.6-0.8$ \\
\hline
\end{tabular}

Measurements are expressed in pounds

- for the masseter contraction force, participants were asked to exert the maximum dental intercuspation with the masseter-specific probe plate in contact with the masseter muscle. The same procedure was performed for both masseter muscles.

\section{Statistical analisys}

Mean values and standard deviations were carried out using Statistica Software 12 (StatSoft $\AA$, TIBCO ${ }^{\circledR}$ Software Inc, Palo Alto, CA, USA). Differences between groups were analysed using a $t$-test with the P-value set at $<0.05$.

\section{Results}

As showed in Figure 1, we found a significant difference $(\mathrm{P}=0.02)$ between $\mathrm{OG}$ and $\mathrm{NO}-\mathrm{OG}$ for the tongue extension force, whereas no significant differences $(\mathrm{P}>0.05)$ were found between groups for the lip pressure and contraction force of both masseters. In particular, as reported in Table 2, the NO-OG showed lower mean value of tongue extension force compared to the reference measurements, whereas the OG was exactly at the minimum value of the normal range. As concern the
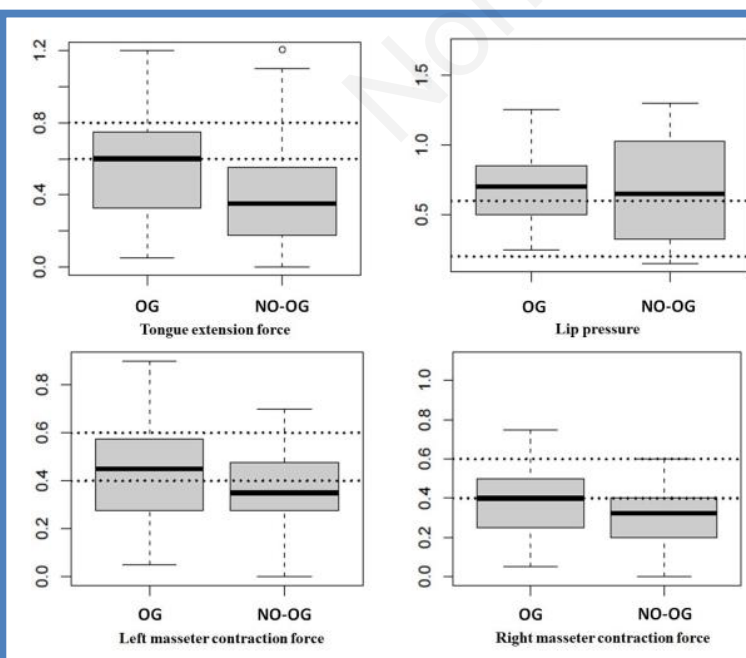

Fig 1. Measurements, expressed in pounds, of perioral muscles forces of the orthodontic group $(O G)$ and the no orthodontic group (NO-OG). masseter contraction force, the OG showed mean values of both masseter muscles within the physiological range, whereas the NO-OG showed lower strength levels for the masseter muscles, left and right alike. Regarding the lip pressure, the OG and the NO-OG showed similar and dysfunctional levels compared to the reference values.

\section{Discussion}

Swallowing dysfunction is a frequent disorder among children and refers to an altered tongue posture and abnormal tongue movement during swallowing. ${ }^{11,12}$ This atypical mechanism of swallowing determines an imbalance muscles of the cranio-cervical-mandibular system and establishes posture adaptations and, moreover, can influences fitness characteristics. ${ }^{26,27}$ To normalize this physiological function, removable functional appliance is one of the treatments applied by dentistry. ${ }^{19}$ The purpose of this study was to measure orofacial muscles activity in children aged 6-10 years with (OG) and without (NO-OG) removable functional appliance. Our results showed a significant difference on tongue extension force between groups. In particular, the mean value of the $\mathrm{OG}$ coincided exactly with the minimum reference level of the normal range, whereas the NO-OG showed lower mean value of tongue extension force. Our findings are in agreement with clinicians and researchers reporting an enhancement of neuromuscular activity of orofacial muscles in patients with functional appliances..$^{21}$ Moreover, a biomechanical reason related to the change in perioral muscle activity is the modification of mandible position during functional orthodontic treatment due to the direction of the intraoral traction forces exerted by the functional appliance that induces to a different basal muscle tone. ${ }^{19,28,29}$ Orthodontic functional appliances rebalance orofacial muscle activity that resulted altered in oral dysfunction prior the treatment particularly in children in which facial growth is ongoing. ${ }^{19,30}$ As to masseter contraction force, group comparisons for both masseter muscles showed contraction force values within the normal range for the OG and out of the range for the NO-OG, but without statistical significance. Our results are in accord with the findings by Miralles et al. that recorded temporal and masseter muscles activity in children with malocclusion 
Table 2. Measurements of perioral muscles forces

\begin{tabular}{lccc}
\hline & OG & NO-OG & P-value \\
\cline { 2 - 4 } Tongue extension force & $0.58 \pm 0.29$ & $0.41 \pm 0.29$ & 0.02 \\
Lip pressure & $0.70 \pm 0.30$ & $0.70 \pm 0.37$ & n.s. \\
Left masseter contraction & $0.43 \pm 0.21$ & $0.35 \pm 0.19$ & n.s. \\
Right masseter contraction & $0.40 \pm 0.22$ & $0.31 \pm 0.17$ & n.s. \\
\hline
\end{tabular}

OG: orthodontic group; NO-OG: no orthodontic group. Measurements are expressed in pounds

with and without functional appliance reporting significantly higher values of these muscles activity during swallowing. ${ }^{21}$ Regarding the lip pressure, the OG and the NO-OG showed similar and dysfunctional levels compared to the reference values. Our results are supported by Saccucci et al. in which the authors found no differences in orbicular oris muscles contractility in children with malocclusion treated with orthodontics compared to a control group. ${ }^{31}$ Since surface EMG is influenced by many factors as individual's physiology, among the methods existing in order to measure orofacial activity, we used myoscan analysis because it is simpler to apply in children. ${ }^{4,32}$ In this study we enrolled children with removable functional appliance because fixed functional appliance per se is not removable and would not have allowed to evaluate masticatory muscles, in particular for the tongue extension force measurement. However, a limit of our study is to have not distinguished the types of participant's removable functional appliances.

In summary, although our findings suggest that children with swallowing dysfunction and removable functional appliance show orofacial muscles activity within the range of reference values (except for the lip pressure), we hypothesize that, based on the scientific literature, the orthodontic treatment can achieve more effective results with the integration of myofunctional therapy, an orofacial method of muscle exercises used to rebalance perioral muscles and the functions of the stomatognathic system. ${ }^{12,33,34}$ On the basis of our results, we need further studies in order to confirm the present findings and to verify our hypothesis.

\section{List of acronyms}

NO-OG - no-orthodontic group

$\mathrm{OG}$ - orthodontic group

\section{Authors contributions}

All authors have participated in the conception and study design, data acquisition, analysis and interpretation, drafting of the manuscript and revised critically it. All authors have read and approved the final version of the manuscript and agree with the order of presentation of the authors.

\section{Acknowledgments}

The authors would like to thank the participants of the study.

Funding None.

\section{Conflict of Interest}

The authors declare no conflicts of interest.

\section{Ethical Publication Statement}

We confirm that we have read the Journal's position on issues involved in ethical publication and affirm that this report is consistent with those guidelines.

\section{Corresponding Author}

Valerio Giustino, Via Giovanni Pascoli, 6, Palermo, Italia. Tel: +3909123896910 Fax: +3909123860894

Email: valerio.giustino@unipa.it

\section{E-mails of co-authors}

Giuseppe Messina: giuseppe.messina17@unipa.it Francesco Martines: francesco.martines@unipa.it Serena Rizzo: rizzo.sere@gmail.com Alessio Pirino: alessiopirino@yahoo.it Fabio Scoppa: posturologia@chinesis.org

\section{References}

1. Hiraki K, Yamada Y, Kurose M, et al. Application of a barometer for assessment of oral functions: Donders space. J Oral Rehabil 2017;44:65-72.

2. Messina G. The Tongue, Mandible, Hyoid System. Eur J Transl Myol 2017;27(1):6363.

3. Tardieu C, Dumitrescu M, Giraudeau A, et al. Dental occlusion and postural control in adults. Neurosci Lett 2009;450:221-4.

4. Patti A, Bianco A, Messina G, et al. The influence of the stomatognathic system on explosive strength: a pilot study. J Phys Ther Sci 2016;28:72-5.

5. Battaglia G, Giustino V, Iovane A, et al. Influence of Occlusal Vertical Dimension on Cervical Spine Mobility in Sports Subjects. Acta Medica Mediterr 2016;32:1589-95.

6. An JS, Jeon DM, Jung WS, et al. Influence of temporomandibular joint disc displacement on 


\section{Swallowing dysfunction and removable functional appliances}

Eur J Transl Myol 29 (3): 246-250, 2019

craniocervical posture and hyoid bone position. Am J Orthod Dentofacial Orthop 2015;147:72-9.

7. Tecco S, Tete S, D'Attilio $M$, et al. Surface electromyographic patterns of masticatory, neck, and trunk muscles in temporomandibular joint dysfunction patients undergoing anterior repositioning splint therapy. Eur $\mathbf{J}$ Orthod 2008;30:592-7.

8. Lee JH. Effects of forward head posture on static and dynamic balance control. J Phys Ther Sci 2016;28:274-7.

9. Cuccia A, Caradonna C. The relationship between the stomatognathic system and body posture. Clinics (Sao Paulo) 2009;64:61-6.

10. López-Soto LM, López-Soto OP, Osorio-Forero A, Restrepo F, Tamayo-Orrego L. Muscle Activity and Muscle Strength in Atypical Swallowing. Salud Uninorte 2017;33:273-84.

11. Kim BR, Sung IY, Choi KH, et al. Long-term outcomes in children with swallowing dysfunction. Dev Neurorehabil 2014;17:298-305.

12. Saccomanno S, Di Tullio A, D'Alatri L, et al. Proposal for a myofunctional therapy protocol in case of altered lingual frenulum. A pilot study. Eur J Paediatr Dent 2019;20:67-72.

13. Pancherz H. Activity of the temporal and masseter muscles in class II, division 1 malocclusions. An electromyographic investigation. Am J Orthod 1980;77:679-88.

14. Wozniak K, Piatkowska D, Lipski M, et al. Surface electromyography in orthodontics - a literature review. Med Sci Monit 2013;19:416-23.

15. Sumonsiri P, Thongudomporn U. Surface Electromyographic Studies on Masticatory Muscle Activity Related to Orthodontics: A Review of Literature. J Dent Assoc Thai 2017;67(2):107-18.

16. Kiliaridis S. Masticatory muscle influence on craniofacial growth. Acta Odontol Scand 1995;53:196-202.

17. Van Dyck C, Dekeyser A, Vantricht E, et al. The effect of orofacial myofunctional treatment in children with anterior open bite and tongue dysfunction: a pilot study. Eur J Orthod 2016;38:227-34.

18. Horn H, Kuhnast K, Axmann-Krcmar D, et al. Influence of orofacial dysfunctions on spatial and temporal dimensions of swallowing movements. J Orofac Orthop 2004;65:376-88.

19. DiBiase AT, Cobourne MT, Lee RT. The use of functional appliances in contemporary orthodontic practice. Br Dent J 2015;218:123-8.

20. Yamin-Lacouture C, Woodside DG, Sectakof PA, et al. The action of three types of functional appliances on the activity of the masticatory muscles. Am J Orthod Dentofacial Orthop 1997;112:560-72.
21. Miralles R, Berger B, Bull R, et al. Influence of the activator on electromyographic activity of mandibular elevator muscles. Am J Orthod Dentofacial Orthop 1988;94:97-103.

22. Wozniak K, Piatkowska D, Szyszka-Sommerfeld L, et al. Impact of functional appliances on muscle activity: a surface electromyography study in children. Med Sci Monit 2015;21:246-53.

23. Angle EH. Classification of Malocclusion. The Dental Cosmos 1899;41:248-64.

24. Hanson ML. Orofacial myofunctional disorders: guidelines for assessment and treatment. Int $\mathbf{J}$ Orofacial Myology 1988;14:27-32.

25. Garliner D. Myofunctional therapy. Gen Dent 1976;24:30-40.

26. Battaglia G, Messina G, Giustino V, et al. Influence of Vertical Dimension of Occlusion on Peak Force During Handgrip Tests in Athletes. Asian J Sports Med 2018;9:e68274.

27. Maurer C, Heller S, Sure JJ, et al. Strength improvements through occlusal splints? The effects of different lower jaw positions on maximal isometric force production and performance in different jumping types. PLoS One 2018;13:e0193540.

28. McNamara JA, Jr. Neuromuscular and skeletal adaptations to altered function in the orofacial region. Am J Orthod 1973;64:578-606.

29. Carels C, van Steenberghe D. Changes in neuromuscular reflexes in the masseter muscles during functional jaw orthopedic treatment in children. Am J Orthod Dentofacial Orthop 1986;90:410-9.

30. Di Palma E, Tepedino M, Chimenti C, et al. Effects of the functional orthopaedic therapy on masticatory muscles activity. J Clin Exp Dent 2017;9:e886-e91.

31. Saccucci M, Tecco S, Ierardoa G, et al. Effects of interceptive orthodontics on orbicular muscle activity: a surface electromyographic study in children. J Electromyogr Kinesiol 2011;21:665-71.

32. Suvinen TI, Malmberg J, Forster C, et al. Postural and dynamic masseter and anterior temporalis muscle EMG repeatability in serial assessments. J Oral Rehabil 2009;36:814-20.

33. de Felicio CM, Melchior Mde O, Ferreira CL, et al. Otologic symptoms of temporomandibular disorder and effect of orofacial myofunctional therapy. Cranio 2008;26:118-25.

34. Homem MA, Vieira-Andrade RG, Falci SG, et al. Effectiveness of orofacial myofunctional therapy in orthodontic patients: a systematic review. Dental Press J Orthod 2014;19:94-9.

Submission: May, 7, 2019

Revision received: June 3, 2019

Acceptance: June 5, 2019 